\title{
Rapid analgesia for prehospital hip disruption (RAPID): protocol for feasibility study of randomised controlled trial
}

\author{
Jenna K. Bulger ${ }^{1 *}$, Alan Brown², Bridie A. Evans', Greg Fegan', Simon Ford ${ }^{3}$, Katy Guy ${ }^{3}$, Sian Jones², Leigh Keen ${ }^{4}$, \\ Ashrafunnesa Khanom ${ }^{1}$, Ian Pallister ${ }^{3}$, Nigel Rees ${ }^{4}$, Ian T. Russell ${ }^{1}$, Anne C. Seagrove ${ }^{1}$ and Helen A. Snooks ${ }^{1}$
}

\begin{abstract}
Background: Adequate pain relief at the point of injury and during transport to hospital is a major challenge in all acute traumas, especially for those with hip fractures, whose injuries are difficult to immobilise and whose long-term outcomes may be adversely affected by administration of opiate analgesics. Fascia iliaca compartment block (FICB) is a procedure routinely undertaken by doctors and nurses in the emergency department for patients with hip fracture but not yet evaluated for use by paramedics at the scene of emergency calls.

In this feasibility study, we aim to test whether FICB administered by paramedics at the scene of participants' hip fractures is feasible, safe and acceptable. This will enable us to decide whether to proceed to a fully powered, multi-centre pragmatic randomised trial to evaluate whether the procedure is effective for patients and worthwhile for the NHS.

Methods/design: In this study, we propose to recruit ten paramedics in an urban area of South Wales. We will train them to carry out FICB when they attend patients with hip fracture. We will randomly allocate eligible patients to FICB or usual care using audited scratch cards. We will follow up participants to assess measurability of key outcomes including quality of life, pain scores, adverse events, length of stay in hospital, acceptability to patients and compliance of paramedics. We will assess whether the findings meet specified feasibility criteria and, if so, plan a full trial.
\end{abstract}

Discussion: This study will enable us to recommend whether to undertake a definitive trial of FICB by paramedics for hip fracture.

Trial registration: ISRCTN60065373

Keywords: Paramedic, Hip fracture, Fractured neck of femur, Analgesia, Prehospital, Pain

\section{Background}

It is predicted that 6.3 million hip fractures a year will occur worldwide by 2050 [1]. In the UK, hip fractures result in more admissions to orthopaedic trauma wards than patients with any other injury [2]; this has a huge financial impact on the National Health Service (NHS) [3].

Hip fracture is associated with a high mortality rate (30\% at 1 year). Delay to surgery-over $48 \mathrm{~h}$-has a detrimental effect on patient mortality [4-6]. Early surgery has also been shown to reduce the incidence of postoperative pneumonia (relative risk 0.59) and pressure

\footnotetext{
*Correspondence: j.k.bulger@swansea.ac.uk

${ }^{1}$ Swansea University Medical School, ILS2, Singleton Campus, Swansea

University, SA2 8PP Swansea, UK

Full list of author information is available at the end of the article
}

sores (relative risk 0.48) [7]. The National Institute for Health and Care Excellence [8] therefore advises that surgery should take place on the day of admission or the following day.

Prehospital management of patients with hip fracture can cause severe pain as the injury site is difficult to immobilise and ambulance staff must move the patient by lifting and negotiating through obstacles such as stairs and doorways. Paramedics have a range of available pain relief options including paracetamol, opiates and Entonox, with the most frequently administered being intravenous (IV) morphine [9]. However, several studies have suggested that prehospital pain relief for patients with suspected hip fracture is inadequate, with up to $40 \%$ of patients not receiving any pain relief at all 
[10-13]. Following interviews with patients, who believed their prehospital care could be improved, Aronsson et al. [14] recommended that alternative methods of pain relief should be considered before admission to hospital.

Unfortunately, the pharmacokinetics and pharmacodynamics of opiates mean that unpredictable responses can occur, particularly in the elderly; adverse drug effects when morphine is administered are therefore likely and have the potential to delay a patient being taken to theatre [15]. Rainer et al. [16] showed that IV ketorolac was more cost-effective than IV morphine in isolated limb injury; odds of an adverse event with IV morphine were 144 times (95\% confidence interval from 41 to 502) more likely. Alternatives to opiate-based pain relief are therefore desirable in the care of hip fracture.

The prehospital management of hip fractures is vital to provide adequate pain relief, ideally without potential side effects that may delay surgical fixation and have a detrimental effect on patient outcomes. We hypothesise that the use of fascia iliaca compartment block (FICB) to provide pain relief to patients with a broken hip before they are taken to the emergency department (ED) may improve the outcome for patients with hip fracture in this way.

FICB is increasingly used in ED and orthopaedic wards in the care of patients with hip fracture. The technique was first described by Dalens et al. in 1989 [17]. Lees et al. [18] demonstrated statistically significantly reduced pain scores in patients receiving FICB in hospital, compared with controls. Lengths of stay and mortality rates were also reduced, although there may have been confounding variables [18]. Increasingly, the evidence in the literature suggests that FICB is easy to learn, provides better pain relief and triggers fewer side effects than opiates [19-26].

The Association of Anaesthetists of Great Britain and Ireland supports FICB delivered by non-physician practitioners when a physician is not immediately available [27]. The Association states that non-medical registered health professionals can perform FICB if they have been appropriately trained and are following agreed clinical governance procedures.

\section{Feasibility study aim}

The aim of the study is to assess the feasibility of undertaking a fully powered, multi-centre pragmatic randomised trial to test the clinical and cost-effectiveness of paramedics providing FICB as early pain relief for patients who have fractured a hip at the scene of their injury.

\section{Feasibility study objectives}

To assess:
1. Accuracy of recognition of hip fracture by paramedics and thus the safety and feasibility of FICB

2. Willingness of both patients and paramedics to participate in the study

3. Compliance with the FICB protocol by paramedics

4. Sample size required for a full randomised controlled trial (RCT) and recruitment period required to achieve this target

5. Acceptability of FICB as method of providing pain relief in prehospital care of patients with hip fracture

6. Which outcome measures to use in a full RCT and at what point: for example, pain scores before and after pain relief; whether the administration of FICB in prehospital care yields benefits for patients besides pain relief, notably side effects of opioids (nausea, constipation, respiratory depression and confusion); length of time before surgery; and length of stay in hospital. We shall also assess the ability of participants to complete forms, the incidence of missing data and the time taken to complete data collection

7. Whether study processes and outcomes achieve specified feasibility criteria for trial implementation

\section{Methods/design}

\section{Design}

Single-centre randomised parallel-group feasibility trial.

\section{Setting}

The scenes of patients' injuries, in the predominantly urban catchment area of one ED in South Wales, where the average job cycle time is $90 \mathrm{~min}$.

\section{Paramedic participants}

We have recruited ten paramedics based at ambulance stations in that catchment area and trained them in FICB.

\section{Inclusion criteria}

Adult patients (aged 18 years or older) who are:

- Attended out of hospital by a participating emergency paramedic following a 999 call

- Assessed by the attending paramedic as having an isolated hip fracture; conscious (Glasgow Coma Scale score at least 13) and haemodynamically stable

- Conveyed to the participating hospital

We exclude patients if they refuse analgesia or if the emergency paramedic is working alone without back-up from the advanced paramedic practitioner, emergency medical technician or other paramedic.

\section{Sample size}

In this randomised feasibility study, we are not seeking to evaluate FICB and have not therefore undertaken a 
formal power calculation. However, considering that the participating hospital treated about 370 patients with hip fracture over 12 months in 2013-2014, and accounting for shift patterns of paramedics and the number of patients excluded for other reasons, we estimate that ten trained paramedics can recruit 50 eligible patients into the trial over 12 months-enough to assess whether the trial meets our specified feasibility criteria for progressing to a full trial.

\section{Intervention}

\section{Paramedic training}

Our proposed training reflects published methods for training non-medical healthcare professionals $[28,29]$ and expert advice from consultants in anaesthetics and trauma surgery who have previously trained nurses to carry out FICB in the ED. We train paramedics through an online package including a video showing the administration of FICB, followed by group sessions led by a consultant anaesthetist (SF). Pairs of paramedics then attend sessions at the participating hospital where they administer FICB to real patients observed by an anaesthetist. They alternate between administering and critiquing the FICB to ensure their learning is active [30]. The paramedics must pass a competency assessment before they can recruit patients to the study.

The paramedics will attend a 6-month refresher halfway through the year of patient recruitment. We advise them to contact the paramedic research support officer if they feel they need additional training or support during the recruitment period, typically from the consultant anaesthetist.

Our paramedics routinely measure pain scores on a numerical rating scale, a standard tool used by healthcare professionals. The patient clinical record used by paramedics already has a designated box to collect these data as a standard part of the patient's assessment. We therefore provide no additional training for recording pain scores within this trial.

\section{Treatment protocol}

We give paramedics a printed treatment protocol as an aide mémoire during recruitment. Should a patient be randomly allocated to receive $\mathrm{FICB}$, the paramedic will ensure the participant has no contraindication to FICB including allergy to local anaesthetic; use of anticoagulants; neurovascular damage to the affected leg; previous femoral bypass surgery; infection at the site of injection; inability to palpate the femoral artery on the affected leg; hip prosthesis on the affected side; pregnancy; or body mass apparently less than $50 \mathrm{~kg}$.

\section{Usual care}

At present, paramedics provide a range of analgesia to patients with hip fracture, including IV morphine, paracetamol and Entonox [10]. Participants allocated at random to the control group receive this usual pain relief, as judged appropriate by the paramedic. To participants in the intervention group, paramedics can offer paracetamol and Entonox in addition to FICB. We advise paramedics not to give morphine for at least $20 \mathrm{~min}$ after patients have received FICB. If FICB has not relieved the pain after $20 \mathrm{~min}$, however, they can give morphine if they judge that appropriate.

\section{Randomisation}

Before recruiting participants, we produced 100 sequentially numbered scratch cards with concealed trial allocations generated by the trial statistician (GF). To avoid subversion of the randomisation procedure, in particular tampering with the scratch cards, we check that study paramedics use these scratch cards for eligible patients in sequence and account for them on their ambulance stations' randomisation log. We regularly audit these logs against the remaining scratch cards.

\section{Patient consent}

We take consent in two stages:

1. Consent to treatment: paramedics obtain oral consent to treatment (i.e. analgesia) according to usual practise, for example for cannulation or venepuncture.

2. Consent to participation in the trial: An NHS researcher seeks retrospective consent from patients to take part in the trial within ten working days of their injury. This usually occurs in hospital, or in the community if necessary. For patients with cognitive impairment, we seek consent to take part in the trial from relatives or carers. Thus, we recognise that it is not ethically appropriate to consent patients to research in a medical emergency [31]. Indeed, in our SAFER randomised trials in emergency care [32, 33], we gained ethical information governance and research approvals to inform patients of their potential inclusion in research within 10 days of attendance by emergency ambulance. The PARAMEDIC trial used similar of enrolling patients, using a waiver of consent under the Mental Capacity Act, and asking patients for consent to follow up after this [34]. Consistent with those trials, all patients who dissent from RAPID then leave the study.

\section{Study flowchart}

All study paramedics keep a laminated copy of the study flowchart as an aide mémoire (Fig. 1).

\section{Outcomes}

We are testing the following outcome measures for the full RCT: 


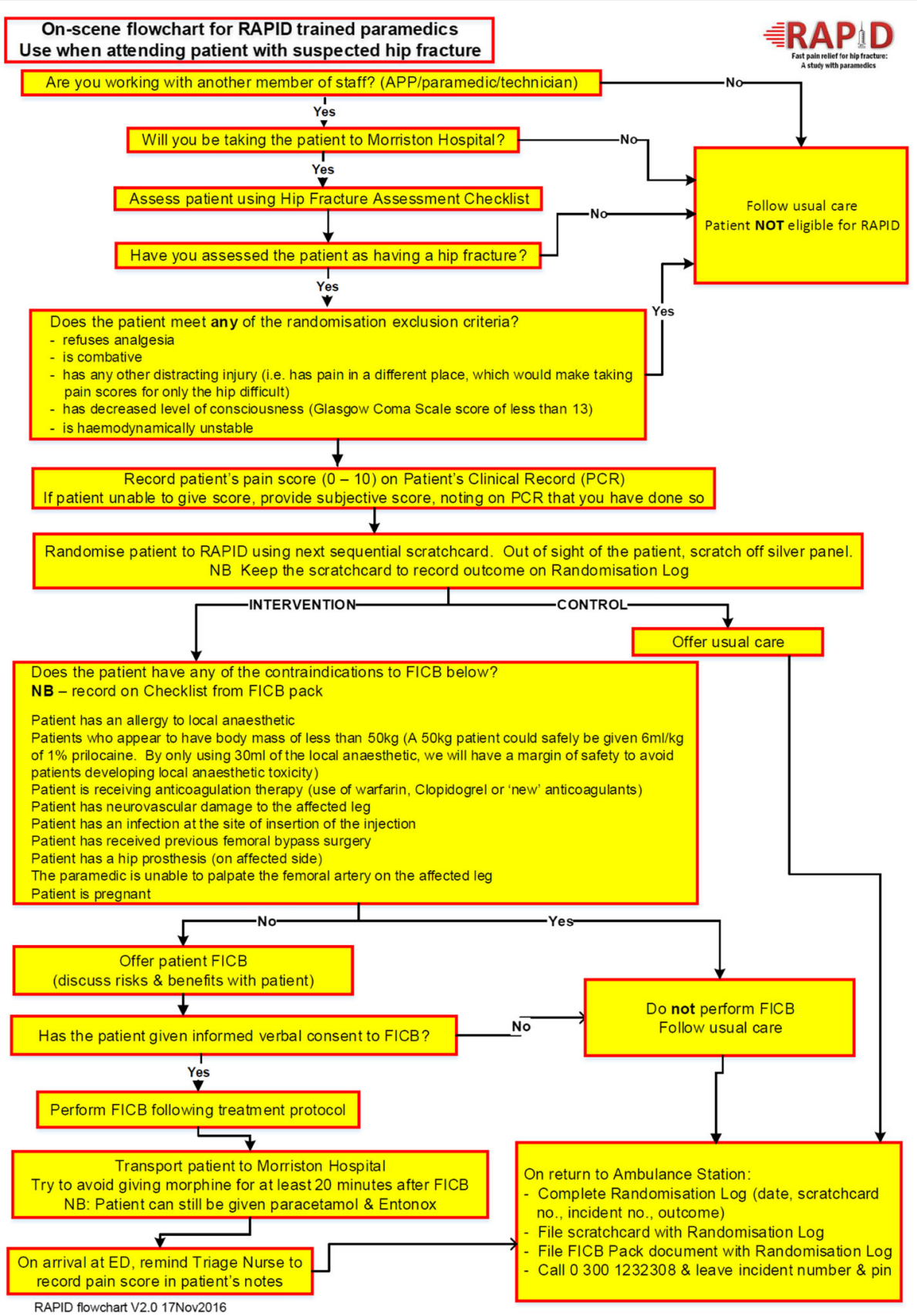

Fig. 1 Study flowchart

\section{Primary}

Self-reported:

- Health-related quality of life, using SF-12 [35] at 30 days and 6 months

Routinely collected:

- Mortality at 6 months

\section{Secondary}

Self-reported:

- Mobility score, using the Rivermead Mobility Index at 30 days and 6 months [36]

- Initial pain score at the scene of participants' injuries before pain relief, using an 11-point numeric rating scale with 0 showing no pain at all and 10 showing the worst pain imaginable [37] 
- Satisfaction with care received from paramedics using a modified Quality of Care Monitor [38] 30 days after the participant's injury

Routinely collected:

- Length of inpatient stay

- Pain score on arrival in the ED using the same 11-point numeric rating scale [37]

- Participant safety assessed by adverse events (AEs) occurring in each trial arm, notably their severity, causality and expectedness

- Duration of paramedic's management of participant ('job cycle time')

- Use of anti-emetics and alternative analgesia

- Time between arrival at ED and surgery

\section{Qualitative data collection}

We will interview ten intervention participants to explore their experiences of receiving FICB at the scene of their injury. This will enable us to explore whether this method of providing analgesia for hip fracture is acceptable to patients (Study Objective 5). We will sample respondents purposively [39] to ensure that they cover the range of the following characteristics:

- Age

- Gender

- Previous hip fracture

- Time of injury

- Study paramedic who treated the participant

We shall record and transcribe these interviews.

Towards the end of the recruitment period, we will also conduct paramedic focus groups by inviting all ten trained paramedics to take part. We will explore their experiences of randomising participants and providing FICB at the scene of injury (Study Objectives 2 and 3) and ask how this could work on a larger scale, to aid our planning of a fully powered RCT.

\section{Data management}

We use an electronic data capture and management system designed for clinical trials, to store anonymised data securely [40]. We use participants' contact details only to arrange interviews and send postal questionnaires and store them on a password-protected computer separate from the trial database. Transcripts of qualitative interviews and focus groups will have participants' study number but no identifiable information. We shall also store digital recordings on a passwordprotected computer.

\section{Analysis}

Quantitative

Quantitative analysis will enable us to assess our data against the following feasibility criteria [41], which we seek to meet within reasonable limits:

1. Recruit at least ten paramedics to conduct the trial

2. Paramedics recognise hip fracture with sensitivity of $75 \%$ and positive predictive value of $85 \%$

3. At least $50 \%$ of intervention participants receive the intervention

4. At least $60 \%$ of recruited participants consent to follow up

5. Retrieve primary outcomes for at least $70 \%$ of consented participants

6. Mean participant satisfaction in intervention group is at least $80 \%$ of that in control group

7. Clinicians are in equipoise about safety and effectiveness of paramedic-administered FICB

8. Balance of serious AEs between groups

We base the criteria for progression on direct measures of a count (criterion 1) and direct measures of proportions (criteria 3-6). We will use receiver operating characteristic analysis to generate the positive predictive value (criterion 2) with the first X-ray providing the gold standard.

To judge whether criterion 7 has been met, we shall compare health-related quality of life, mobility, satisfaction with care and change in pain scores following analgesia using $t$ tests of mean scores, mortality risk ratios, median lengths of inpatient stay, paramedic job cycle time and time between arrival at ED and surgery using a Wilcoxon non-parametric test. These exploratory analyses will also enable us to estimate the sample size for a full trial.

We shall report the results of this feasibility trial according to the CONSORT guidelines [42].

\section{Qualitative}

We will conduct thematic analysis of data from interviews and focus groups. This is a systematic and transparent method of analysis which generates themes from the implicit and explicit ideas contained in the original accounts of participants. One researcher will lead the analysis. Two others, including a lay representative, will independently monitor key stages of coding, generating themes and interpretation and adopt a critical stance to test and confirm findings [43-45].

\section{Lay contribution}

Lay representatives with experience and knowledge of hip fractures and emergency care contributed to developing the research plan, drafting the application for funding and preparing all documentation, notably the Patient Information Sheet. Two lay representatives will 
sit on the Trial Management Group and two others on the Trial Steering Committee throughout the trial, to present patients' perspectives and play a full part in interpretation, reporting and dissemination of findings. We follow best practise in supporting their contribution so they collaborate as equal members of the study team throughout $[46,47]$.

\section{Discussion}

Given the nature of the intervention, it is not possible to blind paramedics or participants to the treatment they receive; sham FICB would be unethical. To reduce the risk of bias in reporting and analysing pain scores, paramedics record participants' baseline pain scores before randomisation. The entire research team except the data manager will remain blind to participants' allocations until the Trial Steering Committee has approved the primary analysis.

Variable paramedic compliance with the protocol is a danger. Hence, we shall monitor the following: use of the randomisation scratch cards to prevent subversion; missed recruitment of eligible participants; and compliance with study allocation to prevent contamination.

As we randomise participants before they consent, there is potential for biassed groups if more participants consent to follow up in one arm of the feasibility study than the other. Though we do not expect such an imbalance, the data we collect will allow us to assess this risk.

As this feasibility trial has requested volunteers to take part, they are not necessarily a representative sample of all the paramedics in the area; we acknowledge that this is a limitation. If we recommend a fully powered trial of paramedic-provided FICB, we shall invite all paramedics in the study areas to take part.

We shall compare outcomes between groups only to ensure that there are no large differences between groups and that we remain in equipoise about the clinical effectiveness of prehospital paramedic-administered FICB for hip fracture. We do not aim to evaluate clinical effectiveness in this feasibility study. Hence, we will interpret observed differences in outcomes between groups with caution in this underpowered feasibility study.

The findings of this feasibility study will enable us to recommend whether to conduct a definitive RCT of FICB by paramedics for hip fracture. If so, we shall prepare for a fully powered multi-centre trial, notably by liaising with other ambulance services and EDs to engage further sites; and drafting a proposal for research funding to evaluate whether FICB is clinically effective for patients and cost-effective for the NHS.

\section{Trial status}

Ongoing: participant recruitment has commenced.

\section{Abbreviations}

AE: Adverse event; ED: Emergency department; FICB: Fascia iliaca compartment block; IV: Intravenous; NHS: National Health Service; RCT: Randomised controlled trial; WAST: Welsh Ambulance Services NHS Trust

\section{Funding}

The Health and Care Research Wales, part of the Welsh Government, has funded RAPID through their Research for Patient and Public Benefit (RFPPB) stream (1003)

\section{Availability of data and materials}

Data sharing not applicable to this article as no datasets have yet been generated or analysed during the current feasibility study.

\section{Authors' contributions}

JKB and ACS drafted the manuscript with editorial input from all the authors. NR IP and SF conceived and developed the research with methodological advice from GF, HAS and ITR. All authors read and approved the final manuscript.

\section{Authors' information}

$\mathrm{JKB}$ is a junior doctor and research officer at Swansea University, who is undertaking a PhD about prehospital analgesia for patients with hip fracture. ACS, BAE and AK are research officers at Swansea University. JKB and ACS are the trial managers for RAPID, with AK providing the maternity leave cover for JKB. AB and SJ represent the lay representatives on the Trial Management Group. GF is a professor of clinical trials and director of Swansea Trials Unit. $\mathrm{SF}$ is a consultant anaesthetist in Swansea. KG is a consultant of emergency medicine in Swansea. IP is a professor of trauma and orthopaedics in Swansea. NR is the head of research and innovation for the Welsh Ambulance Services NHS

Trust and chief investigator for RAPID. LK is the paramedic research support officer for RAPID. ITR is an emeritus professor of clinical trials at Swansea University. HAS is a professor of Health Services Research at Swansea University.

\section{Competing interests}

The authors declare that they have no competing interests.

\section{Ethics approval}

The Wales Research Ethics Committee 6 granted ethical approval (reference 15/WA/0439).

\section{Author details}

'Swansea University Medical School, ILS2, Singleton Campus, Swansea University, SA2 8PP Swansea, UK. ${ }^{2}$ Patient Representatives, Swansea, UK. ${ }^{3}$ Abertawe Bro Morgannwg University Health Board, Swansea, UK. ${ }^{4}$ Welsh Ambulance Services NHS Trust, Swansea, UK.

Received: 4 March 2016 Accepted: 8 December 2016

Published online: 23 January 2017

\section{References}

1. Friedman SM, Mendelson DA. Epidemiology of fragility fractures. Clin Geriatr Med. 2014:30:175-81.

2. National Institute for Health and Care Excellence. Hip Fracture in Adults Quality Standard [QS16]. 2012. http://www.nice.org.uk/guidance/qs16. Accessed 5 Jan 2017.

3. Lawrence TM, White CT, Wenn R, Moran CG. The current hospital costs of treating hip fractures. Injury. 2005;36:88-92.

4. Dy CJ, McCollister KE, Lubarsky DA, Lane JM. An economic evaluation of a systems-based strategy to expedite surgical treatment of hip fractures. J Bone Joint Surg. 2011;93:1326-34.

5. Moran CG, Wenn R, Sikand M, Taylor AM. Early mortality after hip fracture: is delay before surgery important? J Bone Joint Surg. 2005;87:483-9.

6. Holt G, Smith R, Duncan K, McKeown DW. Does delay to theatre for medical reasons affect the peri-operative mortality in patients with a fracture of the hip? J Bone Joint Surg. 2010;92:835-41.

7. Simunovic N, Devereaux PJ, Sprague S, Guyatt GH, Schemitsch E, Debeer J, et al. Effect of early surgery after hip fracture on mortality and complications: systematic review and metaanalysis. CMAJ. 2010;182:1609-16.

8. National Institute for Health and Care Excellence (NICE). Hip fracture: the management of hip fracture in adults. 2014. https:/www.nice.org.uk/ Guidance/CG124. Accessed 5 Jan 2017. 
9. Association of Ambulance Chief Executives \& Joint Royal Colleges Ambulance Liaison Committee. UK Ambulance Services Clinical Practice Guidelines 2013. Class Publishing, Bridgwater.

10. Holdgate A, Shepherd SA, Huckson S. Patterns of analgesia for fractured neck of femur in Australian emergency departments. Emerg Med Australas. 2010;22:3-8.

11. McEachin CC, McDermott JT, Swor R. Few emergency medical services patients with lower-extremity fractures receive prehospital analgesia. Prehospital Emergency Care. 2002;6:406-10.

12. Simpson PM, Bendall JC, Tiedemann A, Lord SR, Close JC. Provision of outof-hospital analgesia to older fallers with suspected fractures: above par, but opportunities for improvement exist. Acad Emerg Med. 2013;20:761-8.

13. Vassiliadis J, Hitos K, Hill CT. Factors influencing prehospital and emergency department analgesia administration to patients with femoral neck fractures. Emerg Med (Fremantle). 2002;14:261-6.

14. Aronsson K, Bjorkdahl I, Wireklint SB. Prehospital emergency care for patients with suspected hip fractures after falling - older patients' experiences. J Clin Nurs. 2014;23:3115-23.

15. Chau DL, Walker V, Pai L, Cho LM. Opiates and elderly: use and side effects. Clin Interv Aging. 2008;3:273-8

16. Rainer TH, Jacobs P, Ng YC, Cheung NK, Tam M, Lam PKW, et al. Cost effectiveness analysis of intravenous ketorolac and morphine for treating pain after limb injury: double blind randomised controlled trial. BMJ. 2000; 321:1247-51.

17. Dalens B, Vanneuville G, Tanguy A. Comparison of the fascia iliaca compartment block with the 3-in-1 block in children. Anesth Analg. 1989; 69:705-13.

18. Lees D, Harrison WD, Ankers T, A'Court J, Marriott A, Shipsey D, et al. Fascia iliaca compartment block for hip fractures: experience of integrating a new protocol across two hospital sites. Eur J Emerg Med. 2014. doi:10.1097/MEJ. 0000000000000167.

19. About-Setta AM, Beaupre LA, Rashiq S, Dryden DM, Hamm MP, Sadowski $C A$, et al. Comparative effectiveness of pain management interventions for hip fracture: a systematic review. Ann Intern Med. 2011;155:234-45.

20. Godoy Monzon D, Vazquez J, Jauregui JR, Iserson KV. Pain treatment in post-traumatic hip fracture in the elderly: regional block vs. systemic nonsteroidal analgesics. Int J Emerg Med. 2010;3:321-5.

21. Hogh A, Dremstrup L, Jensen SS, Lindholt J. Fascia iliaca compartment block performed by junior registrars as a supplement to pre-operative analgesia for patients with hip fracture. StratTraum Limb Recon. 2008:3:65-70.

22. Mouzopoulos G, Vasiliadis G, Lasanianos N, Nikolaras G, Morakis E, Kaminaris M. Fascia iliaca block prophylaxis for hip fracture patients at risk for delirium: a randomized placebo controlled study. J Orthop Traumatol. 2009;10:127-33.

23. Obideyi A, Srikantharajah I, Grigg L, Randall A. Nurse administered fascia iliaca compartment block for pre-operative pain relief in adult fractured neck of femur. Acute Pain. 2008;10:145-9.

24. Randall A, Grigg L, Obideyi A, Srikantharajah I. Fascia iliaca compartment block: a nurse-led initiative for preoperative pain management in patients with a fractured neck of femur. J Orthop Nurs. 2008:12:69-74.

25. Rashid A, Beswick E, Fitton L, Galitzine S. Regional analgesia in the emergency department for hip fractures: survey of current UK practice and its impact on developing the service in a teaching hospital: 8AP4-5. Eur J Anaesthesiol. 2012;29:125.

26. Rawal N, Allvin R. Acute pain services in Europe: a 17-nation survey of 105 hospitals. The EuroPain Acute Pain Working Party. Eur J Anaesthesiol. 1998; 15:354-63.

27. Association of Anaesthetists of Great Britain and Ireland. Fascia Iliaca Blocks and Non-Physician Practitioners. London: AAGNl; 2013. http://www.aagbi. org/sites/default/files/Fascia\%20llaica\%20statement\%2022JAN2013.pdf. Accessed 5 Jan 2017.

28. Dochez E, van Geffen GJ, Bruhn J, Hoogerwerf N, van de Pas H, Scheffer G. Prehospital administered fascia iliaca compartment block by emergency medical service nurses, a feasibility study. Scand J Trauma Resusc Emerg Med. 2014;22:38

29. MCRae PJ, Bendall JC, Madigan V, Middleton PM. Paramedic-performed fascia iliaca compartment block for femoral fractures: a controlled trial. J Emerg Med. 2015;48:581-9.

30. Wolff M, Wagner MJ, Poznanski S, Schiller J, Santen S. Not another boring lecture: engaging learners with active learning techniques. J Emerg Med. 2015:48:85-93.
31. General Medical Council. Good practice in Research and Consent to Research. London: GMC; 2010. http://www.gmcuk.org/Good_practice_in research_and_consent_to_research.pdf_58834843.pdf. Accessed 5 Jan 2017.

32. Snooks H, Cheung WY, Close J, Dale J, Gaze S, Humphreys I, et al. Support and Assessment for Fall Emergency Referrals (SAFER 1) trial protocol. Computerised on-scene decision support for emergency ambulance staff to assess and plan care for older people who have fallen: evaluation of costs and benefits using a pragmatic cluster randomised trial. BMC Emerg Med. 2010;10:2.

33. Snooks H, Anthony R, Chatters R, Cheung WY, Dale J, Donohoe R, et al. Support and assessment for fall emergency referrals (SAFER 2) research protocol: cluster randomised trial of the clinical and cost effectiveness of new protocols for emergency ambulance paramedics to assess and refer to appropriate community-based care. BMJ Open. 2012;2:e002169.

34. Perkins GD, Lall R, Quinn T, Deakin CD, Cooke MW, Horton J, et al. Mechanical versus manual chest compression for out-of-hospital cardiac arrest (PARAMEDIC): a pragmatic, cluster randomised controlled trial. Lancet. 2015;385(9972):947-55.

35. Hurst NP, Ruta DA and Kind P. Comparison of the MOS short form-12 (SF12) health status questionnaire with the SF36 in patients with rheumatoid arthritis. Br J Rheumatol. 1998;37(8):862-9.

36. Collen FM, Wade DT, Robb GF, Bradshaw CM. The Rivermead Mobility Index: a further development of the Rivermead Motor Assessment. Int Disabil Stud. 1991;13(2):50-4.

37. Paice JA, Cohen FL. Validity of a verbally administered numeric rating scale to measure cancer pain intensity. Cancer Nurs. 1997;20(2):88-93.

38. Carey RG, Seibert JH. A patient survey system to measure quality improvement: questionnaire reliability and validity. Med Care. 1993;31:834-45.

39. Barbour RS. Checklists for improving rigour in qualitative research: a case of the tail wagging the dog? BMJ. 2001;322:1115.

40. https://www.elsevier.com/solutions/InferMed/edc-in-clinical-research. Accessed 5 Jan 2017.

41. Charlesworth G, Burnell K, Hoe J, Orrell M, Russell I. Acceptance checklist for clinical effectiveness pilot trials: a systematic approach. BMC Med Res Methodol. 2013;13:78.

42. Schulz KF, Altman DG, Moher D, CONSORT Group. CONSORT 2010 Statement: updated guidelines for reporting parallel group randomised trials. PLoS Med. 2010;7(3):e1000251.

43. Marshall C, Rossman G. Designing qualitative research. 5th ed. Los Angeles: Sage; 2011.

44. Ziebland S, McPherson A. Making sense of qualitative data analysis: an introduction with illustrations from DIPEx (personal experiences of health and illness). Med Educ. 2006;40:405-14.

45. Miles MB, Huberman AM. Qualitative data analysis: an expanded sourcebook 2nd ed. London: Sage; 1994.

46. Evans BA, Bedson E, Bell P, Hutchings H, Lowes L, Rea D, et al. Involving service users in trials: developing a standard operating procedure. Trials. 2013;14:219.

47. INVOLVE. Briefing notes for researchers: involving the public in NHS, public health and social care research. Eastleigh: INVOLVE; 2012.

\section{Submit your next manuscript to BioMed Central and we will help you at every step:}

- We accept pre-submission inquiries

- Our selector tool helps you to find the most relevant journal

- We provide round the clock customer support

- Convenient online submission

- Thorough peer review

- Inclusion in PubMed and all major indexing services

- Maximum visibility for your research

Submit your manuscript at www.biomedcentral.com/submit 